\title{
STRONGLY NONLINEAR ELLIPTIC VARIATIONAL UNILATERAL PROBLEMS IN ORLICZ SPACE
}

\author{
L. AHAROUCH, A. BENKIRANE, AND M. RHOUDAF
}

Received 2 March 2005; Accepted 12 April 2005

We will be concerned with the existence result of unilateral problem associated to the equations of the form $A u+g(x, u, \nabla u)=f$, where $A$ is a Leray-Lions operator from its domain $D(A) \subset W_{0}^{1} L_{M}(\Omega)$ into $W^{-1} E_{\bar{M}}(\Omega)$. On the nonlinear lower order term $g(x, u$, $\nabla u)$, we assume that it is a Carathéodory function having natural growth with respect to $|\nabla u|$, and satisfies the sign condition. The right-hand side $f$ belongs to $W^{-1} E_{\bar{M}}(\Omega)$.

Copyright (c) $2006 \mathrm{~L}$. Aharouch et al. This is an open access article distributed under the Creative Commons Attribution License, which permits unrestricted use, distribution, and reproduction in any medium, provided the original work is properly cited.

\section{Introduction}

Let $\Omega$ be an open bounded subset of $\mathbb{R}^{N}, N \geq 2$, with segment property. Let us consider the following nonlinear Dirichlet problem:

$$
-\operatorname{div}(a(x, u, \nabla u))+g(x, u, \nabla u)=f
$$

$A u=-\operatorname{div} a(x, u, \nabla u)$ is a Leray-Lions operator defined on its domain $\mathscr{D}(A) \subset$ $W_{0}^{1} L_{M}(\Omega)$, with $M$ an $N$-function and where $g$ is a nonlinearity with the "natural" growth condition

$$
|g(x, s, \xi)| \leq b(|s|)(h(x)+M(|\xi|))
$$

and which satisfies the classical sign condition

$$
g(x, s, \xi) \cdot s \geq 0
$$

The right-hand side $f$ belongs to $W^{-1} E_{\bar{M}}(\Omega)$. 
An existence theorem has been proved in [15] where the nonlinearity $g$ depend only on $x$ and $u$, and in [3] where $g$ depends also on the $\nabla u$ but the author's suppose the $\Delta_{2}$-condition, while in [8] the author's were concerned of the above problem without assuming a $\Delta_{2}$-condition on $M$.

It is our purpose, in this paper, to prove an existence result for unilateral problems associated to (1.1) without assuming the $\Delta_{2}$-condition.

In our paper, the mean difficulty is the second and the third steps where we study the a priori estimate. To overcome this difficulty, we have changed the classical coercivity condition by the following one:

$$
a(x, s, \zeta)\left(\zeta-\nabla v_{0}\right) \geq \alpha M(|\zeta|)-\delta(x) \quad \text { see }\left(\mathrm{A}_{4}\right) \text { below, }
$$

(this idea is inspired from the work [16]).

Note that in the case of the equation, the a priori estimate is easily proved in [8] thanks to the some classical technique (see [16]).

Furthermore, in our work, we have not supposed any regularity assumption on the obstacle. Note that this type of equations can be applied in sciences physics. Non-standard examples of $M(t)$ which occur in the mechanics of solids and fluids are $M(t)=t \log (1+$ $t), M(t)=\int_{0}^{t} s^{1-\alpha}(\operatorname{arcsinh})^{\alpha} d s(0 \leq \alpha \leq 1)$ and $M(t)=t \log (1+\log (1+t))$ (see $[10,11$, $13,12]$ for more details).

This paper is organized as follows. Section 2 contains some preliminaries and some technical lemmas. Section 3 is concerned with basic assumptions and the main result which is proved in Section 4.

\section{Preliminaries}

2.1. Let $M: \mathbb{R}^{+} \rightarrow \mathbb{R}^{+}$be an $N$-function, that is, $M$ is continuous, convex, with $M(t)>0$ for $t>0, M(t) / t \rightarrow 0$ as $t \rightarrow 0$ and $M(t) / t \rightarrow \infty$ as $t \rightarrow \infty$.

Equivalently, $M$ admits the representation: $M(t)=\int_{0}^{t} a(s) d s$ where $a: \mathbb{R}^{+} \rightarrow \mathbb{R}^{+}$is nondecreasing, right continuous, with $a(0)=0, a(t)>0$ for $t>0$ and $a(t)$ tends to $\infty$ as $t \rightarrow \infty$.

The $N$-function $\bar{M}$ conjugate to $M$ is defined by $\bar{M}=\int_{0}^{t} \bar{a}(s) d s$, where $\bar{a}: \mathbb{R}^{+} \rightarrow \mathbb{R}^{+}$is given by $\bar{a}(t)=\sup \{s: a(s) \leq t\}$.

The $N$-function $M$ is said to satisfy the $\Delta_{2}$-condition if, for some $k$

$$
M(2 t) \leq k M(t) \quad \forall t \geq 0 .
$$

When (2.1) holds only for $t \geq$ some $t_{0}>0$, then $M$ is said to satisfy the $\Delta_{2}$-condition near infinity.

We will extend these $N$-functions even functions on all $\mathbb{R}$.

Moreover, we have the following Young's inequality:

$$
\forall s, t \geq 0, \quad s t \leq M(t)+\bar{M}(s) .
$$

Let $P$ and $Q$ be two $N$-functions. $P \ll Q$ means that $P$ grows essentially less rapidly than $Q$, that is, for each $\epsilon>0, P(t) / Q(\epsilon t) \rightarrow 0$ as $t \rightarrow \infty$.

This is the case if and only if $\lim _{t \rightarrow \infty}\left(Q^{-1}(t) / P^{-1}(t)\right)=0$. 
2.2. Let $\Omega$ be an open subset of $\mathbb{R}^{N}$. The Orlicz class $K_{M}(\Omega)$ (resp., the Orlicz space $L_{M}(\Omega)$ ) is defined as the set of (equivalence classes of) real valued measurable functions $u$ on $\Omega$ such that

$$
\int_{\Omega} M(u(x)) d x<+\infty \quad\left(\text { resp., } \int_{\Omega} M\left(\frac{u(x)}{\lambda}\right) d x<+\infty \text { for some } \lambda>0\right) .
$$

$L_{M}(\Omega)$ is a Banach space under the norm

$$
\|u\|_{M, \Omega}=\inf \left\{\lambda>0, \int_{\Omega} M\left(\frac{u(x)}{\lambda}\right) d x \leq 1\right\}
$$

and $K_{M}(\Omega)$ is a convex subset of $L_{M}(\Omega)$.

The closure in $L_{M}(\Omega)$ of the set of bounded measurable functions with compact support in $\bar{\Omega}$ is denoted by $E_{M}(\Omega)$.

The dual of $E_{M}(\Omega)$ can be identified with $L_{\bar{M}}(\Omega)$ by means of the pairing $\int_{\Omega} u v d x$, and the dual norm of $L_{\bar{M}}(\Omega)$ is equivalent to $\|\cdot\|_{\bar{M}, \Omega}$.

2.3. We now turn to the Orlicz-Sobolev space, $W^{1} L_{M}(\Omega)$ (resp., $W^{1} E_{M}(\Omega)$ ) is the space of all functions $u$ such that $u$ and its distributional derivatives of order 1 lie in $L_{M}(\Omega)$ (resp., $E_{M}(\Omega)$ ). It is a Banach space under the norm

$$
\|u\|_{1, M}=\sum_{|\alpha| \leq 1}\left\|D^{\alpha} u\right\|_{M}
$$

Thus, $W^{1} L_{M}(\Omega)$ and $W^{1} E_{M}(\Omega)$ can be identified with subspaces of the product of $N+$ 1 copies of $L_{M}(\Omega)$. Denoting this product by $\Pi L_{M}$, we will use the weak topologies $\sigma\left(\Pi L_{M}, \Pi E_{\bar{M}}\right)$ and $\sigma\left(\Pi L_{M}, \Pi L_{\bar{M}}\right)$.

The space $W_{0}^{1} E_{M}(\Omega)$ is defined as the (norm) closure of the Schwartz space $D(\Omega)$ in $W^{1} E_{M}(\Omega)$ and the space $W_{0}^{1} L_{M}(\Omega)$ as the $\sigma\left(\Pi L_{M}, \Pi E_{\bar{M}}\right)$ closure of $D(\Omega)$ in $W^{1} L_{M}(\Omega)$.

2.4. Let $W^{-1} L_{\bar{M}}(\Omega)$ (resp., $W^{-1} E_{\bar{M}}(\Omega)$ ) denote the space of distributions on $\Omega$ which can be written as sums of derivatives of order $\leq 1$ of functions in $L_{\bar{M}}(\Omega)$ (resp., $E_{\bar{M}}(\Omega)$ ). It is a Banach space under the usual quotient norm (for more details see [1]).

We recall some lemmas introduced in [3] which will be used later.

Lemma 2.1. Let $F: \mathbb{R} \rightarrow \mathbb{R}$ be uniformly Lipschitzian, with $F(0)=0$. Let $M$ be an $N$-function and let $u \in W^{1} L_{M}(\Omega)$ (resp., $W^{1} E_{M}(\Omega)$ ). Then $F(u) \in W^{1} L_{M}(\Omega)$ (resp., $W^{1} E_{M}(\Omega)$ ). Moreover, if the set $D$ of discontinuity points of $F^{\prime}$ is finite, then

$$
\frac{\partial}{\partial x_{i}} F(u)= \begin{cases}F^{\prime}(u) \frac{\partial}{\partial x_{i}} u & \text { a.e. in }\{x \in \Omega: u(x) \notin D\}, \\ 0 & \text { a.e. in }\{x \in \Omega: u(x) \in D\} .\end{cases}
$$

Lemma 2.2. Let $F: \mathbb{R} \rightarrow \mathbb{R}$ be uniformly Lipschitzian, with $F(0)=0$. We assume that the set of discontinuity points of $F^{\prime}$ is finite. Let $M$ be an $N$-function, then the mapping $F$ : $W^{1} L_{M}(\Omega) \rightarrow W^{1} L_{M}(\Omega)$ is sequentially continuous with respect to the weak ${ }^{*}$ topology $\sigma\left(\Pi L_{M}, \Pi E_{\bar{M}}\right)$. 
We give now the following lemma which concerns operators of Nemytskii type in Orlicz spaces (see [3]).

LeMma 2.3. Let $\Omega$ be an open subset of $\mathbb{R}^{N}$ with finite measure.

Let $M, P$ and $Q$ be $N$-functions such that $Q \ll P$, and let $f: \Omega \times \mathbb{R} \rightarrow \mathbb{R}$ be a Carathéodory function such that, for a.e. $x \in \Omega$ and all $s \in \mathbb{R}$ :

$$
|f(x, s)| \leq c(x)+k_{1} P^{-1} M\left(k_{2}|s|\right),
$$

where $k_{1}, k_{2}$ are real constants and $c(x) \in E_{Q}(\Omega)$.

Then the Nemytskii operator $N_{f}$ defined by $N_{f}(u)(x)=f(x, u(x))$ is strongly continuous from $\mathscr{P}\left(E_{M}(\Omega), 1 / k_{2}\right)=\left\{u \in L_{M}(\Omega): d\left(u, E_{M}(\Omega)\right)<1 / k_{2}\right\}$ into $E_{Q}(\Omega)$.

Below, we will use the following technical lemma.

Lemma 2.4. Let $\left(f_{n}\right), f \in L^{1}(\Omega)$ such that

(i) $f_{n} \geq 0$ a.e. in $\Omega$,

(ii) $f_{n} \rightarrow f$ a.e. in $\Omega$,

(iii) $\int_{\Omega} f_{n}(x) d x \rightarrow \int_{\Omega} f(x) d x$,

then $f_{n} \rightarrow f$ strongly in $L^{1}(\Omega)$.

\section{Main results}

Let $\Omega$ be an open bounded subset of $\mathbb{R}^{N}, N \geq 2$, with the segment property.

Given an obstacle function $\psi: \Omega \rightarrow \mathbb{R}$, we consider

$$
K_{\psi}=\left\{u \in W_{0}^{1} L_{M}(\Omega) ; u \geq \psi \text { a.e. in } \Omega\right\}
$$

this convex set is sequentially $\sigma\left(\Pi L_{M}, \Pi E_{\bar{M}}\right)$ closed in $W_{0}{ }^{1} L_{M}(\Omega)$. (See [16].) We now state conditions on the differential operator

$$
A u=-\operatorname{div}(a(x, u, \nabla u))
$$

$\left(\mathrm{A}_{1}\right) a(x, s, \xi): \Omega \times \mathbb{R} \times \mathbb{R}^{N} \rightarrow \mathbb{R}^{N}$ is a Carathéodory function.

$\left(\mathrm{A}_{2}\right)$ There exist two $N$-functions $M$ and $P$ with $P \ll M$, function $c(x)$ in $E_{\bar{M}}(\Omega)$, constant $k_{1}, k_{2}, k_{3}, k_{4}$ such that, for a.e. $x$ in $\Omega$ for all $s, \zeta \in \mathbb{R}$

$$
|a(x, s, \zeta)| \leq c(x)+k_{1} \bar{P}^{-1} M\left(k_{2}|s|\right)+k_{3} \bar{M}^{-1} M\left(k_{4}|\zeta|\right) .
$$

$\left(\mathrm{A}_{3}\right)\left[a(x, s, \zeta)-a\left(x, s, \zeta^{\prime}\right)\right]\left(\zeta-\zeta^{\prime}\right)>0$ for a.e. $x$ in $\Omega, s$ in $\mathbb{R}$ and $\zeta^{\prime}$ in $\mathbb{R}^{N}$, with $\zeta \neq \zeta^{\prime}$.

$\left(\mathrm{A}_{4}\right)$ There exists $\delta(x)$ in $L^{1}(\Omega)$ and a strictly positive constant $\alpha$ such that, for some fixed element $v_{0}$ in $K_{\psi} \cap W_{0}^{1} E_{M}(\Omega) \cap L^{\infty}(\Omega)$.

$$
a(x, s, \zeta)\left(\zeta-D v_{0}\right) \geq \alpha M(|\zeta|)-\delta(x)
$$

for a.e. $x$ in $\Omega, s \in \mathbb{R}$ and all $\zeta \in \mathbb{R}^{N}$.

$\left(\mathrm{A}_{5}\right)$ For each $v \in K_{\psi} \cap L^{\infty}(\Omega)$ there exists a sequence $v_{j} \in K_{\psi} \cap W_{0}^{1} E_{M}(\Omega) \cap L^{\infty}(\Omega)$ such that $v_{j} \rightarrow v$ for the modular convergence. 
Furthermore let $g: \Omega \times \mathbb{R} \times \mathbb{R}^{N} \rightarrow \mathbb{R}$ be a Carathéodory function such that for a.e. $x \in \Omega$ and for all $s \in \mathbb{R}$ and all $\zeta \in \mathbb{R}^{N}$.

$\left(\mathrm{G}_{1}\right) g(x, s, \zeta) s \geq 0$

$\left(\mathrm{G}_{2}\right)|g(x, s, \zeta)| \leq b(|s|)(h(x)+M(|\zeta|))$,

where $b: \mathbb{R}_{+} \rightarrow \mathbb{R}_{+}$is continuous non decreasing function, $h$ is given nonnegative function in $L^{1}(\Omega)$.

We define, for $s$ and $k$ in $\mathbb{R}, k \geq 0 T_{k}(s)=\max (-k, \min (k, s))$.

Consider the following Dirichlet problem:

$$
A(u)+g(x, u, \nabla u)=f \quad \text { in } \Omega .
$$

Remark 3.1. Remark that the condition $\left(\mathrm{A}_{5}\right)$ is holds if the one of the following conditions is verified.

(i) There exist $\bar{\psi} \in K_{\psi}$ such that $\psi-\bar{\psi}$ is continuous in $\Omega$ (see [16, Proposition 9]).

(ii) $\psi \in W_{0}^{1} E_{M}(\Omega)$ (see [16, Proposition 10]).

We will prove the following existence theorem.

Theorem 3.2. Assume that $\left(A_{1}\right)-\left(A_{5}\right),\left(G_{1}\right)$ and $\left(G_{2}\right)$ hold and $f \in W^{-1} E_{\bar{M}}(\Omega)$. Then there exists at least one solution of the following unilateral problem:

$$
\begin{gathered}
u \in K_{\psi}(\Omega), \quad g(x, u, \nabla u) \in L^{1}(\Omega), g(x, u, \nabla u) u \in L^{1}(\Omega), \\
\int_{\Omega} a(x, u, \nabla u) \nabla(u-v) d x+\int_{\Omega} g(x, u, \nabla u)(u-v) d x \\
\leq\langle f, u-v\rangle, \quad \forall v \in K_{\psi} \cap L^{\infty}(\Omega) .
\end{gathered}
$$

Remark 3.3. We remark that the statement of the previous theorem does not exists in the case of Sobolev space. But, some existence result in this sense have been proved under the regularity assumption $\psi^{+} \in W_{0}^{1, p}(\Omega) \cap L^{\infty}(\Omega)$ (see [7]).

Remark 3.4. We recall that, differently from the methods used in $[7,9]$, we do not introduce the function $\psi^{+}$in the test function used in the step of a priori estimate.

\section{Proof of Theorem 3.2}

To prove the existence theorem, we proceed by steps.

Step 1. Approximate unilateral problems.

Let us define

$$
g_{n}(x, s, \xi)=\frac{g(x, s, \xi)}{1+(1 / n)|g(x, s, \xi)|}
$$

and let us consider the approximate unilateral problems:

$$
\begin{gathered}
u_{n} \in K_{\psi} \cap D(A), \\
\left\langle A u_{n}, u_{n}-v\right\rangle+\int_{\Omega} g_{n}\left(x, u_{n}, \nabla u_{n}\right)\left(u_{n}-v\right) d x \leq\left\langle f, u_{n}-v\right\rangle, \\
\forall v \in K_{\psi} .
\end{gathered}
$$


From Gossez and Mustonen [16, Proposition 5], the problem $\left(P_{n}\right)$ has at least one solution.

Step 2. A priori estimates.

Let $k \geq\left\|v_{0}\right\|_{\infty}$ and let $\varphi_{k}(s)=s e^{\gamma s^{2}}$, where $\gamma=(2 b(k) / \alpha)^{2}$.

It is well known that

$$
\varphi_{k}^{\prime}(s)-\frac{b(k)}{\alpha}\left|\varphi_{k}(s)\right| \geq \frac{1}{2}, \quad \forall s \in \mathbb{R}(\text { see }[7])
$$

Since $f \in W^{-1} E_{\bar{M}}(\Omega)$ then $f$ can be written as follows:

$$
f=f_{0}-\operatorname{div} F \quad \text { with } f_{0} \in E_{\bar{M}}(\Omega), F \in\left(E_{\bar{M}}(\Omega)\right)^{N}
$$

Taking $u_{n}-\eta \varphi_{k}\left(T_{l}\left(u_{n}-v_{0}\right)\right)$ as test function in $\left(P_{n}\right)$, where $l=k+\left\|v_{0}\right\|_{\infty}$, we obtain

$$
\begin{gathered}
\int_{\Omega} a\left(x, u_{n}, \nabla u_{n}\right) \nabla T_{l}\left(u_{n}-v_{0}\right) \varphi_{k}^{\prime}\left(T_{l}\left(u_{n}-v_{0}\right)\right) d x+\int_{\Omega} g_{n}\left(x, u_{n}, \nabla u_{n}\right) \varphi_{k}\left(T_{l}\left(u_{n}-v_{0}\right)\right) d x \\
\leq \int_{\Omega} f_{0} \varphi_{k}\left(T_{l}\left(u_{n}-v_{0}\right)\right) d x+\int_{\Omega} F \nabla T_{l}\left(u_{n}-v_{0}\right) \varphi_{k}^{\prime}\left(T_{l}\left(u_{n}-v_{0}\right)\right) d x .
\end{gathered}
$$

Since $g_{n}\left(x, u_{n}, \nabla u_{n}\right) \varphi_{k}\left(T_{l}\left(u_{n}-v_{0}\right)\right) \geq 0$ on the subset $\left\{x \in \Omega:\left|u_{n}(x)\right|>k\right\}$, then

$$
\begin{aligned}
& \int_{\left\{\left|u_{n}-v_{0}\right| \leq l\right\}} a\left(x, u_{n}, \nabla u_{n}\right) \nabla\left(u_{n}-v_{0}\right) \varphi_{k}^{\prime}\left(T_{l}\left(u_{n}-v_{0}\right)\right) d x \\
& \leq \int_{\left\{\left|u_{n}\right| \leq k\right\}}\left|g_{n}\left(x, u_{n}, \nabla u_{n}\right)\right|\left|\varphi_{k}\left(T_{l}\left(u_{n}-v_{0}\right)\right)\right| d x+\int_{\Omega} f_{0} \varphi_{k}\left(T_{l}\left(u_{n}-v_{0}\right)\right) d x \\
& \quad+\int_{\left\{\left|u_{n}-v_{0}\right| \leq l\right\}} F \nabla u_{n} \varphi_{k}^{\prime}\left(T_{l}\left(u_{n}-v_{0}\right)\right) d x-\int_{\left\{\left|u_{n}-v_{0}\right| \leq l\right\}} F \nabla v_{0} \varphi_{k}^{\prime}\left(T_{l}\left(u_{n}-v_{0}\right)\right) d x,
\end{aligned}
$$

by using $\left(A_{4}\right),\left(G_{1}\right)$ and Young's inequality, we have

$$
\begin{aligned}
& \alpha \int_{\left\{\left|u_{n}-v_{0}\right| \leq l\right\}} M\left(\left|\nabla u_{n}\right|\right) \varphi_{k}^{\prime}\left(T_{l}\left(u_{n}-v_{0}\right)\right) d x \\
& \leq b(|k|) \int_{\Omega}\left(h(x)+M\left(\left|\nabla T_{k}\left(u_{n}\right)\right|\right)\right)\left|\varphi_{k}\left(T_{l}\left(u_{n}-v_{0}\right)\right)\right| d x \\
&+\int_{\Omega} \delta(x) \varphi_{k}^{\prime}\left(T_{l}\left(u_{n}-v_{0}\right)\right) d x+\int_{\Omega} f_{0} \varphi_{k}\left(T_{l}\left(u_{n}-v_{0}\right)\right) d x \\
&+\frac{\alpha}{2} \int_{\left\{\left|u_{n}-v_{0}\right| \leq l\right\}} M\left(\left|\nabla u_{n}\right|\right) \varphi_{k}^{\prime}\left(T_{l}\left(u_{n}-v_{0}\right)\right) d x+C_{1}(k),
\end{aligned}
$$


which implies that

$$
\begin{aligned}
\frac{\alpha}{2} \int_{\left\{\left|u_{n}-v_{0}\right| \leq l\right\}} M\left(\left|\nabla u_{n}\right|\right) \varphi_{k}^{\prime}\left(T_{l}\left(u_{n}-v_{0}\right)\right) d x \\
\leq \quad b(|k|) \int_{\Omega}\left(h(x)+M\left(\left|\nabla T_{k}\left(u_{n}\right)\right|\right)\right)\left|\varphi_{k}\left(T_{l}\left(u_{n}-v_{0}\right)\right)\right| d x \\
\quad+\int_{\Omega} \delta(x) \varphi_{k}^{\prime}\left(T_{l}\left(u_{n}-v_{0}\right)\right) d x+\int_{\Omega} f_{0} \varphi_{k}\left(T_{l}\left(u_{n}-v_{0}\right)\right) d x+C_{1}(k) .
\end{aligned}
$$

Since $\left\{x \in \Omega,\left|u_{n}(x)\right| \leq k\right\} \subseteq\left\{x \in \Omega:\left|u_{n}-v_{0}\right| \leq l\right\}$ and the fact that $h, \delta$ and $f_{0} \in L^{1}(\Omega)$, then

$$
\begin{aligned}
\int_{\Omega} M & \left(\left|\nabla T_{k}\left(u_{n}\right)\right|\right) \varphi_{k}^{\prime}\left(T_{l}\left(u_{n}-v_{0}\right)\right) d x \\
& \leq \frac{b(k)}{\alpha} \int_{\Omega} M\left(\left|\nabla T_{k}\left(u_{n}\right)\right|\right)\left|\varphi_{k}\left(T_{l}\left(u_{n}-v_{0}\right)\right)\right| d x+C_{2}(k),
\end{aligned}
$$

which implies that

$$
\int_{\Omega} M\left(\left|\nabla T_{k}\left(u_{n}\right)\right|\right)\left[\varphi_{k}^{\prime}\left(T_{l}\left(u_{n}-v_{0}\right)\right)-\frac{b(k)}{\beta}\left|\varphi_{k}\left(T_{l}\left(u_{n}-v_{0}\right)\right)\right|\right] d x \leq C_{2}(k) .
$$

By using (4.2), we deduce

$$
\int_{\Omega} M\left(\left|\nabla T_{k}\left(u_{n}\right)\right|\right) d x \leq C_{3}(k)
$$

On the other side, taking $v=v_{0}$ as test function in $\left(P_{n}\right)$, we get

$$
\begin{gathered}
\int_{\Omega} a\left(x, u_{n}, \nabla u_{n}\right)\left(\nabla u_{n}-\nabla v_{0}\right) d x+\int_{\Omega} g_{n}\left(x, u_{n}, \nabla u_{n}\right)\left(u_{n}-v_{0}\right) d x \\
\leq \int_{\Omega} f_{0}\left(u_{n}-v_{0}\right) d x+\int_{\Omega} F \nabla\left(u_{n}-v_{0}\right) d x .
\end{gathered}
$$

Let $k>\left\|v_{0}\right\|_{\infty}$, since $g_{n}\left(x, u_{n}, \nabla u_{n}\right)\left(u_{n}-v_{0}\right) \geq 0$ in the subset $\left\{x \in \Omega ;\left|u_{n}(x)\right| \geq k\right\}$, we deduce

$$
\begin{gathered}
\int_{\Omega} a\left(x, u_{n}, \nabla u_{n}\right)\left(\nabla u_{n}-\nabla v_{0}\right) d x+\int_{\left\{\left|u_{n}(x)\right| \leq k\right\}} g_{n}\left(x, u_{n}, \nabla u_{n}\right)\left(u_{n}-v_{0}\right) d x \\
\leq \int_{\Omega} f_{0}\left(u_{n}-v_{0}\right) d x+\int_{\Omega} F \nabla u_{n} d x-\int_{\Omega} F \nabla v_{0} d x,
\end{gathered}
$$

thus, implies that, by using (4.10) and $\left(\mathrm{G}_{2}\right)$

$$
\int_{\Omega} a\left(x, u_{n}, \nabla u_{n}\right)\left(\nabla u_{n}-\nabla v_{0}\right) d x \leq \int_{\Omega} f_{0} u_{n} d x+\int_{\Omega} F \nabla u_{n} d x+C_{4}(k) .
$$


8 Variational unilateral problems

By using [14, Lemma 5.7] and Young's inequality, we deduce

$$
\begin{gathered}
\int_{\Omega} f_{0} u_{n} d x \leq C+\frac{\alpha}{4} \int_{\Omega} M\left(\left|\nabla u_{n}\right|\right) d x, \\
\int_{\Omega} F \nabla u_{n} d x \leq C^{\prime}+\frac{\alpha}{4} \int_{\Omega} M\left(\left|\nabla u_{n}\right|\right) d x .
\end{gathered}
$$

Combining (4.13), (4.14), we get

$$
\int_{\Omega} a\left(x, u_{n}, \nabla u_{n}\right)\left(\nabla u_{n}-\nabla v_{0}\right) d x \leq \frac{\alpha}{4} \int_{\Omega} M\left(\left|\nabla u_{n}\right|\right) d x+\frac{\alpha}{4} \int_{\Omega} M\left(\left|\nabla u_{n}\right|\right) d x+C_{5}(k),
$$

which implies, by using $\left(\mathrm{A}_{4}\right)$

$$
\alpha \int_{\Omega} M\left(\left|\nabla u_{n}\right|\right) d x \leq \frac{\alpha}{2} \int_{\Omega} M\left(\left|\nabla u_{n}\right|\right) d x+C_{6}(k)
$$

hence

$$
\int_{\Omega} M\left(\left|\nabla u_{n}\right|\right) d x \leq C_{7}(k)
$$

Hence $u_{n}$ is bounded in $W_{0}^{1} L_{M}(\Omega)$. So there exists some $u \in W_{0}^{1} L_{M}(\Omega)$ such that

$$
\begin{gathered}
u_{n} \longrightarrow u \quad \text { weakly in } W_{0}^{1} L_{M}(\Omega) \text { for } \sigma\left(\Pi L_{M}, \Pi E_{\bar{M}}\right), \\
u_{n} \longrightarrow u \quad \text { strongly in } E_{M}(\Omega) \text { and a.e. in } \Omega .
\end{gathered}
$$

Step 3. Boundedness of $\left(a\left(x, u_{n}, \nabla u_{n}\right)\right)_{n}$ in $\left(L_{\bar{M}}(\Omega)\right)^{N}$.

Let $w \in\left(E_{M}(\Omega)\right)^{N}$ be arbitrary, by $\left(\mathrm{A}_{3}\right)$ we have

$$
\left(a\left(x, u_{n}, \nabla u_{n}\right)-a\left(x, u_{n}, w\right)\right)\left(\nabla u_{n}-w\right) \geq 0,
$$

this implies that

$$
a\left(x, u_{n}, \nabla u_{n}\right)\left(w-\nabla v_{0}\right) \leq a\left(x, u_{n}, \nabla u_{n}\right)\left(\nabla u_{n}-\nabla v_{0}\right)-a\left(x, u_{n}, w\right)\left(\nabla u_{n}-w\right)
$$

hence,

$$
\begin{aligned}
\int_{\Omega} a\left(x, u_{n}, \nabla u_{n}\right)\left(w-\nabla v_{0}\right) d x \leq & \int_{\Omega} a\left(x, u_{n}, \nabla u_{n}\right)\left(\nabla u_{n}-\nabla v_{0}\right) d x \\
& +\int_{\Omega} a\left(x, u_{n}, w\right)\left(w-\nabla u_{n}\right) d x
\end{aligned}
$$

We claim that

$$
\int_{\Omega} a\left(x, u_{n}, \nabla u_{n}\right)\left(\nabla u_{n}-v_{0}\right) d x \leq C
$$

with $C$ is positive constant. 
L. Aharouch et al. 9

Indeed, if we take $v=v_{0}$ as test function in $\left(P_{n}\right)$, we get

$$
\int_{\Omega} a\left(x, u_{n}, \nabla u_{n}\right)\left(\nabla u_{n}-\nabla v_{0}\right) d x+\int_{\Omega} g_{n}\left(x, u_{n}, \nabla u_{n}\right)\left(u_{n}-v_{0}\right) d x \leq\left\langle f, u_{n}-v_{0}\right\rangle .
$$

Since $g_{n}\left(x, u_{n}, \nabla u_{n}\right)\left(u_{n}-v_{0}\right) \geq 0$ on the subset $\left\{x \in \Omega,\left|u_{n}\right| \geq\left\|v_{0}\right\|_{\infty}\right\}$, which implies

$$
\begin{aligned}
& \int_{\Omega} a\left(x, u_{n}, \nabla u_{n}\right)\left(\nabla u_{n}-\nabla v_{0}\right) d x \\
& \quad \leq b\left(\left\|v_{0}\right\|_{\infty}\right) \int_{\Omega} h(x) d x+b\left(\left\|v_{0}\right\|_{\infty}\right) \int_{\Omega} M\left(\left|\nabla u_{n}\right|\right) d x+\left\langle f, u_{n}-v_{0}\right\rangle .
\end{aligned}
$$

Combining (4.17) and (4.24), we deduce (4.22).

On the other hand, there exists an $N$-function $Q$ such that $M \ll Q$ and the space $W_{0}^{1} L_{M}(\Omega)$ is continuously embedded in $L_{Q}(\Omega)$. Since the sequence $\left\{\nabla u_{n}\right\}$ is bounded in $L_{M}(\Omega)$, we can choose an $\varepsilon>0$ such that $\int_{\Omega} M\left(\varepsilon \nabla u_{n}\right) d x \leq C_{1}$ and $\int_{\Omega} Q\left(\varepsilon u_{n}\right) d x \leq C_{2}$. We have by $\left(\mathrm{A}_{2}\right)\left|a\left(x, u_{n}, w\right)\right| \leq c(x)+k_{1} \bar{M}^{-1} Q\left(\varepsilon u_{n}\right)+k_{3} \bar{M}^{-1} M\left(k_{4} w\right)+C_{\varepsilon}$. When $\lambda$ large enough we obtain

$$
\begin{aligned}
\int_{\Omega} \bar{M}\left(\frac{\left|a\left(x, u_{n}, w\right)\right|}{\lambda}\right) d x \leq & \frac{1}{\lambda} \int_{\Omega} \bar{M}(c(x)) d x+\frac{k_{1}}{\lambda} \int_{\Omega} Q\left(\varepsilon u_{n}\right) d x \\
& +\frac{k_{3}}{\lambda} \int_{\Omega} M\left(k_{4} w\right) d x+\frac{\bar{M}\left(C_{\varepsilon}\right)}{\lambda} \leq C_{3},
\end{aligned}
$$

thus implies that $\int_{\Omega} a\left(x, u_{n}, w\right)\left(w-\nabla u_{n}\right) d x$ is bounded, therefore by using (4.21) and (4.22), we get

$$
\int_{\Omega} a\left(x, u_{n}, \nabla u_{n}\right)\left(w-\nabla v_{0}\right) d x \leq C_{4}
$$

Since $w$ is arbitrary, we deduce $\int_{\Omega} a\left(x, u_{n}, \nabla u_{n}\right) w d x \leq C_{5}$.

Finally by theorem of Banach-Steinhaus, the sequence $a\left(x, u_{n}, \nabla u_{n}\right)$ remains bounded in $L_{\bar{M}}(\Omega)$.

Step 4. Almost everywhere convergence of the gradient.

We fix $k>\left\|v_{0}\right\|_{\infty}$. Let $\Omega_{r}=\left\{x \in \Omega,\left|\nabla T_{k}(u(x))\right| \leq r\right\}$ and denote by $\chi_{r}$ the characteristic function of $\Omega_{r}$. Clearly, $\Omega_{r} \subset \Omega_{r+1}$ and meas $\left(\Omega \backslash \Omega_{r}\right) \rightarrow 0$ as $r \rightarrow \infty$.

Fix $r$ and let $s \geq r$, we have

$$
\begin{aligned}
0 & \leq \int_{\Omega_{r}}\left[a\left(x, T_{k}\left(u_{n}\right), \nabla T_{k}\left(u_{n}\right)\right)-a\left(x, T_{k}\left(u_{n}\right), \nabla T_{k}(u)\right)\right]\left[\nabla T_{k}\left(u_{n}\right)-\nabla T_{k}(u)\right] d x \\
& \leq \int_{\Omega_{s}}\left[a\left(x, T_{k}\left(u_{n}\right), \nabla T_{k}\left(u_{n}\right)\right)-a\left(x, T_{k}\left(u_{n}\right), \nabla T_{k}(u)\right)\right]\left[\nabla T_{k}\left(u_{n}\right)-\nabla T_{k}(u)\right] d x \\
& =\int_{\Omega_{s}}\left[a\left(x, T_{k}\left(u_{n}\right), \nabla T_{k}\left(u_{n}\right)\right)-a\left(x, T_{k}\left(u_{n}\right), \nabla T_{k}(u) \chi_{s}\right)\right]\left[\nabla T_{k}\left(u_{n}\right)-\nabla T_{k}(u) \chi_{s}\right] d x \\
& \leq \int_{\Omega}\left[a\left(x, T_{k}\left(u_{n}\right), \nabla T_{k}\left(u_{n}\right)\right)-a\left(x, T_{k}\left(u_{n}\right), \nabla T_{k}(u) \chi_{s}\right)\right]\left[\nabla T_{k}\left(u_{n}\right)-\nabla T_{k}(u) \chi_{s}\right] d x .
\end{aligned}
$$


By the condition $\left(\mathrm{A}_{5}\right)$ there exists a sequence $v_{j} \in K_{\psi} \cap W_{0}^{1} E_{M}(\Omega) \cap L^{\infty}(\Omega)$ which converges to $T_{k}(u)$ for the modular converge in $W_{0}^{1} L_{M}(\Omega)$.

Here, we define $w_{n, j}=T_{k}\left(u_{n}\right)-T_{k}\left(v_{j}\right), w_{j}=T_{k}(u)-T_{k}\left(v_{j}\right)$.

For $\eta=\exp \left(-4 \gamma k^{2}\right)$, we defined the following function as

$$
v_{n, j}=u_{n}-\eta \varphi_{k}\left(w_{n, j}\right)
$$

By taking $v_{n, j}$ as test functions in $\left(P_{n}\right)$, we get

$$
\left\langle A\left(u_{n}\right), \eta \varphi_{k}\left(w_{n, j}\right)\right\rangle+\int_{\Omega} g_{n}\left(x, u_{n}, \nabla u_{n}\right) \eta \varphi_{k}\left(w_{n, j}\right) d x \leq\left\langle f, \eta \varphi_{k}\left(w_{n, j}\right)\right\rangle .
$$

Since $\eta$ is nonnegative, then

$$
\left\langle A\left(u_{n}\right), \varphi_{k}\left(w_{n, j}\right)\right\rangle+\int_{\Omega} g_{n}\left(x, u_{n}, \nabla u_{n}\right) \varphi_{k}\left(w_{n, j}\right) d x \leq\left\langle f, \varphi_{k}\left(w_{n, j}\right)\right\rangle .
$$

It follows that

$$
\int_{\Omega} a\left(x, u_{n}, \nabla u_{n}\right) \nabla w_{n, j} \varphi_{k}^{\prime}\left(w_{n, j}\right) d x+\int_{\Omega} g_{n}\left(x, u_{n}, \nabla u_{n}\right) \varphi_{k}\left(w_{n, j}\right) d x \leq\left\langle f, \varphi_{k}\left(w_{n, j}\right)\right\rangle .
$$

Denoting by $\epsilon(n, j)$ any quantity such that

$$
\lim _{j \rightarrow+\infty} \lim _{n \rightarrow+\infty} \epsilon(n, j)=0 .
$$

We get, by (4.31),

$$
\begin{gathered}
\int_{\Omega} a\left(x, u_{n}, \nabla u_{n}\right)\left(\nabla T_{k}\left(u_{n}\right)-\nabla T_{k}\left(v_{j}\right)\right) \varphi_{k}^{\prime}\left(w_{n, j}\right) d x \\
\quad+\int_{\Omega} g_{n}\left(x, u_{n}, \nabla u_{n}\right) \varphi_{k}\left(w_{n, j}\right) d x \leq\left\langle f, \varphi_{k}\left(w_{n, j}\right)\right\rangle .
\end{gathered}
$$

In view of $(4.18)$, we have $\varphi_{k}\left(w_{n, j}\right) \rightarrow \varphi_{k}\left(w_{j}\right)$ weakly in $W_{0}^{1} L_{M}(\Omega)$ for $\sigma\left(\Pi L_{M}, \Pi E_{\bar{M}}\right)$ as $n \rightarrow+\infty$, and then

$$
\left\langle f, \varphi_{k}\left(w_{n, j}\right)\right\rangle \longrightarrow\left\langle f, \varphi_{k}\left(w_{j}\right)\right\rangle \text { as } n \longrightarrow+\infty \text {. }
$$

Again, tends $j$ to infinity, we get

$$
\left\langle f, \varphi_{k}\left(w_{j}\right)\right\rangle \longrightarrow 0 \quad \text { as } j \longrightarrow+\infty
$$

Therefore,

$$
\left\langle f, \varphi_{k}\left(w_{n, j}\right)\right\rangle=\epsilon(n, j)
$$


On the set $\left\{x \in \Omega,\left|u_{n}(x)\right|>k\right\}$, we have $g\left(x, u_{n}, \nabla u_{n}\right) \varphi_{k}\left(w_{n, j}\right) \geq 0$, so by $(4.31)$

$$
\begin{array}{r}
\int_{\Omega} a\left(x, u_{n}, \nabla u_{n}\right)\left(\nabla T_{k}\left(u_{n}\right)-\nabla T_{k}\left(v_{j}\right)\right) \varphi_{k}^{\prime}\left(w_{n, j}\right) d x \\
\quad+\int_{\left\{\left|u_{n}\right| \leq k\right\}} g_{n}\left(x, u_{n}, \nabla u_{n}\right) \varphi_{k}\left(w_{n, j}\right) d x \leq \epsilon(n, j) .
\end{array}
$$

Splitting the first integral on the left-hand side of (4.37) where $\left|u_{n}\right| \leq k$ and $\left|u_{n}\right|>k$, we can write

$$
\begin{aligned}
\int_{\Omega} a\left(x, u_{n}, \nabla u_{n}\right) \nabla w_{n, j} \varphi_{k}^{\prime}\left(w_{n, j}\right) d x & \\
\geq & \int_{\Omega} a\left(x, T_{k}\left(u_{n}\right), \nabla T_{k}\left(u_{n}\right)\right)\left[\nabla T_{k}\left(u_{n}\right)-\nabla T_{k}\left(v_{j}\right)\right] \varphi_{k}^{\prime}\left(w_{n, j}\right) d x \\
& \quad-\varphi_{k}^{\prime}(2 k) \int_{\left\{\left|u_{n}\right|>k\right\}}\left|a\left(x, u_{n}, \nabla u_{n}\right)-a\left(x, T_{k}\left(u_{n}\right), 0\right)\right|\left|\nabla T_{k}\left(v_{j}\right)\right| d x .
\end{aligned}
$$

Since $\left|a\left(x, u_{n}, \nabla u_{n}\right)-a\left(x, T_{k}\left(u_{n}\right), 0\right)\right|$ bounded in $L_{\bar{M}}(\Omega)$ there exists a function $h_{k} \in$ $L_{\bar{M}}(\Omega)$ such that $\left|a\left(x, u_{n}, \nabla u_{n}\right)-a\left(x, T_{k}\left(u_{n}\right), 0\right)\right| \rightarrow h_{k}$ for $\sigma\left(Ł_{\bar{M}}, E_{M}\right)$ as $n \rightarrow+\infty$, while $\left|\nabla T_{k}\left(v_{j}\right)\right| \chi_{\left\{\left|u_{n}\right|>k\right\}} \rightarrow\left|\nabla T_{k}\left(v_{j}\right)\right| \chi_{\{|u|>k\}}$ strongly in $E_{M}(\Omega)$, and by the modular convergence of $T_{k}\left(v_{j}\right)$, we deduce that the second term of the right-hand side of (4.38) tends to 0 as $n \rightarrow \infty$ and $j \rightarrow \infty$, hence

$$
\begin{aligned}
& \int_{\Omega} a\left(x, u_{n}, \nabla u_{n}\right) \nabla w_{n, j} \varphi_{k}^{\prime}\left(w_{n, j}\right) d x \\
& \quad \geq \int_{\Omega} a\left(x, T_{k}\left(u_{n}\right), \nabla T_{k}\left(u_{n}\right)\right)\left[\nabla T_{k}\left(u_{n}\right)-\nabla T_{k}\left(v_{j}\right)\right] \varphi_{k}^{\prime}\left(w_{n, j}\right) d x+\varepsilon(n, j),
\end{aligned}
$$

which implies that

$$
\begin{aligned}
\int_{\Omega} a\left(x, u_{n}, \nabla u_{n}\right) \nabla w_{n, j} \varphi_{k}^{\prime}\left(w_{n, j}\right) d x \\
\geq \int_{\Omega}\left[a\left(x, T_{k}\left(u_{n}\right), \nabla T_{k}\left(u_{n}\right)\right)-a\left(x, T_{k}\left(u_{n}\right), \nabla T_{k}\left(v_{j}\right) \chi_{s}^{j}\right)\right] \\
\quad \times\left[\nabla T_{k}\left(u_{n}\right)-\nabla T_{k}\left(v_{j}\right) \chi_{s}^{j}\right] \varphi_{k}^{\prime}\left(w_{n, j}\right) d x \\
+\int_{\Omega} a\left(x, T_{k}\left(u_{n}\right), \nabla T_{k}\left(v_{j}\right) \chi_{s}^{j}\right)\left[\nabla T_{k}\left(u_{n}\right)-\nabla T_{k}\left(v_{j}\right) \chi_{s}^{j}\right] \varphi_{k}^{\prime}\left(w_{n, j}\right) d x \\
\quad-\int_{\Omega \backslash \Omega_{s}^{j}} a\left(x, T_{k}\left(u_{n}\right), \nabla T_{k}\left(u_{n}\right)\right) \nabla T_{k}\left(v_{j}\right) \varphi_{k}^{\prime}\left(w_{n, j}\right) d x+\epsilon(n, j),
\end{aligned}
$$

where $\chi_{s}^{j}$ denotes the characteristic function of the subset $\Omega_{s}^{j}=\left\{x \in \Omega:\left|\nabla T_{k}\left(v_{j}\right)\right| \leq s\right\}$.

Let a function $l_{k} \in\left(L_{\bar{M}}(\Omega)\right)^{N}$ such that $a\left(x, T_{k}\left(u_{n}\right), \nabla T_{k}\left(u_{n}\right)\right) \rightarrow l_{k}$ for $\sigma\left(\Pi L_{\bar{M}}, \Pi E_{M}\right)$, since $\nabla T_{k}\left(v_{j}\right) \chi_{\Omega \backslash \Omega_{s}^{j}} \varphi_{k}^{\prime}\left(w_{n, j}\right)$ tends to $\nabla T_{k}\left(v_{j}\right) \chi_{\Omega \backslash \Omega_{s}^{j}} \varphi_{k}^{\prime}\left(w_{j}\right)$ strongly in $\left(E_{M}(\Omega)\right)^{N}$, the third term of the right-hand side of (4.40) tends to quantity $\int_{\Omega \backslash \Omega_{s}^{j}} l_{k} \nabla T_{k}\left(v_{j}\right) \varphi_{k}^{\prime}\left(w_{j}\right) d x$ as $n$ tend to infinity. 
Letting now $j$ to infinity, by using the modular convergence of $v_{j}$ and

$$
l_{k} \chi_{\Omega \backslash \Omega_{s}^{j}} \chi_{\left\{\left|v_{j}\right| \leq k\right\}} \varphi_{k}^{\prime}\left(w_{j}\right)
$$

we have

$$
\int_{\Omega} l_{k} \nabla T_{k}\left(v_{j}\right) \chi_{\Omega \backslash \Omega_{s}^{j}} \varphi_{k}^{\prime}\left(w_{j}\right) d x \longrightarrow \int_{\Omega \backslash \Omega_{s}} l_{k} \nabla T_{k}(u) \varphi_{k}^{\prime}(0) d x
$$

as $j$ tend to infinity.

Finally

$$
-\int_{\Omega \backslash \Omega_{s}^{j}} a\left(x, T_{k}\left(u_{n}\right), \nabla T_{k}\left(u_{n}\right)\right) \nabla T_{k}\left(v_{j}\right) \varphi_{k}^{\prime}\left(w_{n, j}\right) d x=-\int_{\Omega \backslash \Omega_{s}} l_{k} \nabla T_{k}(u) \varphi_{k}^{\prime}(0) d x+\epsilon(n, j) .
$$

Concerning the second term of the right-hand side of (4.40), since

$$
a\left(x, T_{k}\left(u_{n}\right), \nabla T_{k}\left(v_{j}\right) \chi_{s}^{j}\right) \varphi_{k}^{\prime}\left(w_{n, j}\right) \longrightarrow a\left(x, T_{k}(u), \nabla T_{k}\left(v_{j}\right) \chi_{s}^{j}\right) \varphi_{k}^{\prime}\left(w_{j}\right)
$$

as $n \rightarrow \infty$ in $\left(E_{\bar{M}}(\Omega)\right)^{N}$ by Lemma 2.3 and $\nabla T_{k}\left(u_{n}\right) \rightarrow \nabla T_{k}(u)$ weakly in $\left(L_{M}(\Omega)\right)^{N}$ for $\sigma\left(\Pi L_{M}, \Pi E_{\bar{M}}\right)$.

Consequently, the second term of the right-hand side of (4.40) tends to quantity $\int_{\Omega} a\left(x, T_{k}(u), \nabla T_{k}\left(v_{j}\right) \chi_{s}^{j}\right)\left[\nabla T_{k}(u)-\nabla T_{k}\left(v_{j}\right) \chi_{s}^{j}\right] \varphi_{k}^{\prime}\left(w_{j}\right) d x$ as $n \rightarrow \infty$, moreover letting $j$ to infinity it is easy to see that

$$
\begin{aligned}
\int_{\Omega} a & \left(x, T_{k}(u), \nabla T_{k}\left(v_{j}\right) \chi_{s}^{j}\right)\left[\nabla T_{k}(u)-\nabla T_{k}\left(v_{j}\right) \chi_{s}^{j}\right] \varphi_{k}^{\prime}\left(w_{j}\right) d x \\
& \longrightarrow \int_{\Omega} a\left(x, T_{k}(u), \nabla T_{k}(u) \chi_{s}\right)\left[\nabla T_{k}(u)-\nabla T_{k}(u) \chi_{s}\right] \varphi_{k}^{\prime}(0) d x \\
& =\int_{\Omega \backslash \Omega_{s}} a\left(x, T_{k}(u), 0\right) \nabla T_{k}(u) \varphi_{k}^{\prime}(0) d x .
\end{aligned}
$$

Combining (4.40), (4.43) and (4.45), we get

$$
\begin{aligned}
\int_{\Omega} a\left(x, u_{n}, \nabla u_{n}\right) \nabla w_{n, j} \varphi_{k}^{\prime}\left(w_{n, j}\right) d x \\
\geq \int_{\Omega}\left[a\left(x, T_{k}\left(u_{n}\right), \nabla T_{k}\left(u_{n}\right)\right)-a\left(x, T_{k}\left(u_{n}\right), \nabla T_{k}\left(v_{j}\right) \chi_{s}^{j}\right)\right] \\
\quad \times\left[\nabla T_{k}\left(u_{n}\right)-\nabla T_{k}\left(v_{j}\right) \chi_{s}^{j}\right] \varphi_{k}^{\prime}\left(w_{n, j}\right) d x \\
\quad+\int_{\Omega \backslash \Omega_{s}} l_{k} \nabla T_{k}(u) \varphi_{k}^{\prime}(0) d x \\
\quad+\int_{\Omega \backslash \Omega_{s}} a\left(x, T_{k}(u), 0\right) \nabla T_{k}(u) \varphi_{k}^{\prime}(0) d x+\epsilon(n, j) .
\end{aligned}
$$


We now return to the second term of the left-hand side of (4.37), we have, by using $\left(\mathrm{A}_{4}\right)$ and $\left(\mathrm{G}_{2}\right)$

$$
\begin{aligned}
&\left|\int_{\left\{\left|u_{n}\right| \leq k\right\}} g_{n}\left(x, u_{n}, \nabla u_{n}\right) \varphi_{k}\left(w_{n, j}\right) d x\right| \\
& \leq b(k) \int_{\Omega}\left(h(x)+M\left(\left|\nabla T_{k}\left(u_{n}\right)\right|\right)\right)\left|\varphi_{k}\left(w_{n, j}\right)\right| d x \\
& \leq b(k) \int_{\Omega} h(x)\left|\varphi_{k}\left(w_{n, j}\right)\right| d x+\frac{b(k)}{\alpha} \int_{\Omega} \delta(x)\left|\varphi_{k}\left(w_{n, j}\right)\right| \\
&+\frac{b(k)}{\alpha} \int_{\Omega} a\left(x, T_{k}\left(u_{n}\right), \nabla T_{k}\left(u_{n}\right)\right) \nabla T_{k}\left(u_{n}\right)\left|\varphi_{k}\left(w_{n, j}\right)\right| d x \\
& \quad-\frac{b(k)}{\alpha} \int_{\Omega} a\left(x, T_{k}\left(u_{n}\right), \nabla T_{k}\left(u_{n}\right)\right) \nabla v_{0}\left|\varphi_{k}\left(w_{n, j}\right)\right| d x \\
& \leq \epsilon(n, j)+\frac{b(k)}{\alpha} \int_{\Omega} a\left(x, T_{k}\left(u_{n}\right), \nabla T_{k}\left(u_{n}\right)\right) \nabla T_{k}\left(u_{n}\right)\left|\varphi_{k}\left(w_{n, j}\right)\right| d x .
\end{aligned}
$$

The last term of the last side of this inequality write as

$$
\begin{aligned}
\frac{b(k)}{\alpha} \int_{\Omega}\left[a\left(x, T_{k}\left(u_{n}\right), \nabla T_{k}\left(u_{n}\right)\right)-a\left(x, T_{k}\left(u_{n}\right), \nabla T_{k}\left(v_{j}\right) \chi_{s}^{j}\right)\right] \\
\quad \times\left[\nabla T_{k}\left(u_{n}\right)-\nabla T_{k}\left(v_{j}\right) \chi_{s}^{j}\right]\left|\varphi_{k}\left(w_{n, j}\right)\right| d x \\
+\frac{b(k)}{\alpha} \int_{\Omega} a\left(x, T_{k}\left(u_{n}\right), \nabla T_{k}\left(v_{j}\right) \chi_{s}^{j}\right)\left[\nabla T_{k}\left(u_{n}\right)-\nabla T_{k}\left(v_{j}\right) \chi_{s}^{j}\right]\left|\varphi_{k}\left(w_{n, j}\right)\right| d x \\
+\frac{b(k)}{\alpha} \int_{\Omega} a\left(x, T_{k}\left(u_{n}\right), \nabla T_{k}\left(u_{n}\right)\right) \nabla T_{k}\left(v_{j}\right) \chi_{s}^{j}\left|\varphi_{k}\left(w_{n, j}\right)\right| d x
\end{aligned}
$$

and reasoning as above, it is easy to see that

$$
\begin{gathered}
\frac{b(k)}{\alpha} \int_{\Omega} a\left(x, T_{k}\left(u_{n}\right), \nabla T_{k}\left(v_{j}\right) \chi_{s}^{j}\right)\left[\nabla T_{k}\left(u_{n}\right)-\nabla T_{k}\left(v_{j}\right) \chi_{s}^{j}\right]\left|\varphi_{k}\left(w_{n, j}\right)\right| d x=\epsilon(n, j), \\
-\frac{b(k)}{\alpha} \int_{\Omega} a\left(x, T_{k}\left(u_{n}\right), \nabla T_{k}\left(u_{n}\right)\right) \nabla T_{k}\left(v_{j}\right) \chi_{s}^{j}\left|\varphi_{k}\left(w_{n, j}\right)\right| d x=\epsilon(n, j) .
\end{gathered}
$$

So that by (4.47) and (4.48) we deduce that

$$
\begin{aligned}
\left|\int_{\left\{\left|u_{n}\right| \leq k\right\}} g_{n}\left(x, u_{n}, \nabla u_{n}\right) \varphi_{k}\left(w_{n, j}\right) d x\right| \\
\leq \frac{b(k)}{\alpha} \int_{\Omega}\left[a\left(x, T_{k}\left(u_{n}\right), \nabla T_{k}\left(u_{n}\right)\right)-a\left(x, T_{k}\left(u_{n}\right), \nabla T_{k}\left(v_{j}\right) \chi_{s}^{j}\right)\right] \\
\times\left[\nabla T_{k}\left(u_{n}\right)-\nabla T_{k}\left(v_{j}\right) \chi_{s}^{j}\right]\left|\varphi_{k}\left(w_{n, j}\right)\right| d x+\epsilon(n, j) .
\end{aligned}
$$


Combining (4.37), (4.46) and (4.50), we obtain

$$
\begin{aligned}
\int_{\Omega}[a & \left.\left(x, T_{k}\left(u_{n}\right), \nabla T_{k}\left(u_{n}\right)\right)-a\left(x, T_{k}\left(u_{n}\right), \nabla T_{k}\left(v_{j}\right) \chi_{s}^{j}\right)\right] \\
& \times\left[\nabla T_{k}\left(u_{n}\right)-\nabla T_{k}\left(v_{j}\right) \chi_{s}^{j}\right] \varphi_{k}^{\prime}\left(w_{n_{i}}\right)-\frac{b(k)}{\alpha}\left|\varphi_{k}\left(w_{n, j}\right)\right| d x \\
\leq & \int_{\Omega \backslash \Omega_{s}} l_{k} \nabla T_{k}(u) \varphi_{k}^{\prime}(0) d x+\int_{\Omega \backslash \Omega_{s}} a\left(x, T_{k}(u), 0\right) \nabla T_{k}(u) \varphi_{k}^{\prime}(0) d x+\epsilon(n, j),
\end{aligned}
$$

which implies that, by using (4.2)

$$
\begin{gathered}
\int_{\Omega}\left[a\left(x, T_{k}\left(u_{n}\right), \nabla T_{k}\left(u_{n}\right)\right)-a\left(x, T_{k}\left(u_{n}\right), \nabla T_{k}\left(v_{j}\right) \chi_{s}^{j}\right)\right] \times\left[\nabla T_{k}\left(u_{n}\right)-\nabla T_{k}\left(v_{j}\right) \chi_{s}^{j}\right] d x \\
\leq 2 \int_{\Omega \backslash \Omega_{s}} l_{k} \nabla T_{k}(u) \varphi_{k}^{\prime}(0) d x+2 \int_{\Omega \backslash \Omega_{s}} a\left(x, T_{k}(u), 0\right) \nabla T_{k}(u) \varphi_{k}^{\prime}(0) d x+\epsilon(n, j) .
\end{gathered}
$$

Now, remark that

$$
\begin{aligned}
& \int_{\Omega}\left[a\left(x, T_{k}\left(u_{n}\right), \nabla T_{k}\left(u_{n}\right)\right)-a\left(x, T_{k}\left(u_{n}\right), \nabla T_{k}(u) \chi_{s}\right)\right]\left[\nabla T_{k}\left(u_{n}\right)-\nabla T_{k}(u) \chi_{s}\right] d x \\
& =\int_{\Omega}\left[a\left(x, T_{k}\left(u_{n}\right), \nabla T_{k}\left(u_{n}\right)\right)-a\left(x, T_{k}\left(u_{n}\right), \nabla T_{k}\left(v_{j}\right) \chi_{s}^{j}\right)\right] \\
& \quad \times\left[\nabla T_{k}\left(u_{n}\right)-\nabla T_{k}\left(v_{j}\right) \chi_{s}^{j}\right] d x \\
& +\int_{\Omega} a\left(x, T_{k}\left(u_{n}\right), \nabla T_{k}\left(v_{j}\right) \chi_{s}^{j}\right)\left[\nabla T_{k}\left(u_{n}\right)-\nabla T_{k}\left(v_{j}\right) \chi_{s}^{j}\right] d x \\
& \quad-\int_{\Omega} a\left(x, T_{k}\left(u_{n}\right), \nabla T_{k}(u) \chi_{s}\right)\left[\nabla T_{k}\left(u_{n}\right)-\nabla T_{k}(u) \chi_{s}\right] d x \\
& +\int_{\Omega} a\left(x, T_{k}\left(u_{n}\right), \nabla T_{k}\left(u_{n}\right)\right)\left[\nabla T_{k}\left(v_{j}\right) \chi_{s}^{j}-\nabla T_{k}(u) \chi_{s}\right] d x .
\end{aligned}
$$

We will pass to the limit in $n$ and $j$ in the last three terms of the right-hand side of the last inequality, we get

$$
\begin{aligned}
& \int_{\Omega} a\left(x, T_{k}\left(u_{n}\right), \nabla T_{k}\left(v_{j}\right) \chi_{s}^{j}\right)\left[\nabla T_{k}\left(u_{n}\right)-\nabla T_{k}\left(v_{j}\right) \chi_{s}^{j}\right] d x \\
& =\int_{\Omega \backslash \Omega_{s}} a\left(x, T_{k}(u), 0\right) \nabla T_{k}(u) d x+\epsilon(n, j), \\
& \int_{\Omega} a\left(x, T_{k}\left(u_{n}\right), \nabla T_{k}(u) \chi_{s}\right)\left[\nabla T_{k}\left(u_{n}\right)-\nabla T_{k}(u) \chi_{s}\right] d x \\
& =\int_{\Omega \backslash \Omega_{s}} a\left(x, T_{k}(u), 0\right) \nabla T_{k}(u) d x+\epsilon(n, j), \\
& \int_{\Omega} a\left(x, T_{k}\left(u_{n}\right), \nabla T_{k}\left(u_{n}\right)\right)\left[\nabla T_{k}\left(v_{j}\right) \chi_{s}^{j}-\nabla T_{k}(u) \chi_{s}\right] d x=\epsilon(n, j),
\end{aligned}
$$


which implies that

$$
\begin{aligned}
\int_{\Omega}\left[a\left(x, T_{k}\left(u_{n}\right), \nabla T_{k}\left(u_{n}\right)\right)-a\left(x, T_{k}\left(u_{n}\right), \nabla T_{k}(u) \chi_{s}\right)\right]\left[\nabla T_{k}\left(u_{n}\right)-\nabla T_{k}(u) \chi_{s}\right] d x \\
=\int_{\Omega}\left[a\left(x, T_{k}\left(u_{n}\right), \nabla T_{k}\left(u_{n}\right)\right)-a\left(x, T_{k}\left(u_{n}\right), \nabla T_{k}\left(v_{j}\right) \chi_{s}^{j}\right)\right] \\
\times\left[\nabla T_{k}\left(u_{n}\right)-\nabla T_{k}\left(v_{j}\right) \chi_{s}^{j}\right] d x+\epsilon(n, j) .
\end{aligned}
$$

Combining (4.27), (4.52) and (4.55), we have

$$
\begin{aligned}
\int_{\Omega_{r}}[ & \left.a\left(x, T_{k}\left(u_{n}\right), \nabla T_{k}\left(u_{n}\right)\right)-a\left(x, T_{k}\left(u_{n}\right), \nabla T_{k}(u)\right)\right]\left[\nabla T_{k}\left(u_{n}\right)-\nabla T_{k}(u)\right] d x \\
& \leq \int_{\Omega}\left[a\left(x, T_{k}\left(u_{n}\right), \nabla T_{k}\left(u_{n}\right)\right)-a\left(x, T_{k}\left(u_{n}\right), \nabla T_{k}(u) \chi_{s}\right)\right]\left[\nabla T_{k}\left(u_{n}\right)-\nabla T_{k}(u) \chi_{s}\right] d x \\
& \leq 2 \int_{\Omega \backslash \Omega_{s}} l_{k} \nabla T_{k}(u) \varphi_{k}^{\prime}(0) d x+2 \int_{\Omega \backslash \Omega_{s}} a\left(x, T_{k}(u), 0\right) \nabla T_{k}(u) \varphi_{k}^{\prime}(0) d x+\epsilon(n, j) .
\end{aligned}
$$

By passing to the limsup over $n$, and letting $j, s$ tend to infinity, we obtain

$$
\lim _{n \rightarrow+\infty} \int_{\Omega_{r}}\left[a\left(x, T_{k}\left(u_{n}\right), \nabla T_{k}\left(u_{n}\right)\right)-a\left(x, T_{k}\left(u_{n}\right), \nabla T_{k}(u)\right)\right]\left[\nabla T_{k}\left(u_{n}\right)-\nabla T_{k}(u)\right] d x=0,
$$

thus implies by the same method used in [4] that

$$
\nabla u \rightarrow \nabla u_{n} \quad \text { a.e. in } \Omega \text {. }
$$

Step 5. Modular convergence of the truncation:

Thanks to (4.58), we have $l_{k}=a\left(x, T_{k}(u), \nabla T_{k}(u)\right)$, which implies by using (4.56)

$$
\begin{aligned}
& \int_{\Omega}\left[a\left(x, T_{k}\left(u_{n}\right), \nabla T_{k}\left(u_{n}\right)\right)\left(\nabla T_{k}\left(u_{n}\right)-\nabla v_{0}\right)+\delta(x)\right] d x \\
& \leq \int_{\Omega}\left[a\left(x, T_{k}\left(u_{n}\right), \nabla T_{k}\left(u_{n}\right)\right)\left(\nabla T_{k}(u) \chi_{s}-\nabla v_{0}\right)+\delta(x)\right] d x \\
& \quad+\int_{\Omega} a\left(x, T_{k}\left(u_{n}\right), \nabla T_{k}(u) \chi_{s}\right)\left(\nabla T_{k}\left(u_{n}\right)-\nabla T_{k}(u) \chi_{s}\right) d x \\
& \quad+2 \int_{\Omega \backslash \Omega_{s}} a\left(x, T_{k}(u), \nabla T_{k}(u)\right) \nabla T_{k}(u) \varphi_{k}^{\prime}(0) d x \\
& \quad+2 \int_{\Omega \backslash \Omega_{s}} a\left(x, T_{k}(u), 0\right) \nabla T_{k}(u) \varphi_{k}^{\prime}(0) d x+\epsilon(n, j),
\end{aligned}
$$


which implies, by using the Fatou's lemma

$$
\begin{aligned}
& \int_{\Omega}\left[a\left(x, T_{k}(u), \nabla T_{k}(u)\right)\left(\nabla T_{k}(u)-\nabla v_{0}\right)+\delta(x)\right] d x \\
& \leq \liminf _{n \rightarrow+\infty} \int_{\Omega}\left[a\left(x, T_{k}\left(u_{n}\right), \nabla T_{k}\left(u_{n}\right)\right)\left(\nabla T_{k}\left(u_{n}\right)-\nabla v_{0}\right)+\delta(x)\right] d x \\
& \leq \limsup _{n \rightarrow+\infty} \int_{\Omega}\left[a\left(x, T_{k}\left(u_{n}\right), \nabla T_{k}\left(u_{n}\right)\right)\left(\nabla T_{k}\left(u_{n}\right)-\nabla v_{0}\right)+\delta(x)\right] d x \\
& \leq \limsup _{n \rightarrow+\infty} \int_{\Omega}\left[a\left(x, T_{k}\left(u_{n}\right), \nabla T_{k}\left(u_{n}\right)\right)\left(\nabla T_{k}(u) \chi_{s}-\nabla v_{0}\right)+\delta(x)\right] d x \\
& \quad+\limsup _{n \rightarrow+\infty} \int_{\Omega} a\left(x, T_{k}\left(u_{n}\right), \nabla T_{k}(u) \chi_{s}\right)\left(\nabla T_{k}\left(u_{n}\right)-\nabla T_{k}(u) \chi_{s}\right) d x \\
& \quad+2 \int_{\Omega \backslash \Omega_{s}} l_{k} \nabla T_{k}(u) \varphi_{k}^{\prime}(0) d x+2 \int_{\Omega \backslash \Omega_{s}} a\left(x, T_{k}(u), 0\right) \nabla T_{k}(u) \varphi_{k}^{\prime}(0) d x+\epsilon(n, j) .
\end{aligned}
$$

Reasoning as above, we have

$$
\begin{gathered}
\limsup _{n \rightarrow+\infty} \int_{\Omega}\left[a\left(x, T_{k}\left(u_{n}\right), \nabla T_{k}\left(u_{n}\right)\right)\left(\nabla T_{k}(u) \chi_{s}-\nabla v_{0}\right)+\delta(x)\right] d x \\
=\int_{\Omega}\left[a\left(x, T_{k}(u), \nabla T_{k}(u)\right)\left(\nabla T_{k}(u) \chi_{s}-\nabla v_{0}\right)+\delta(x)\right] d x, \\
\limsup _{n \rightarrow+\infty} \int_{\Omega} a\left(x, T_{k}\left(u_{n}\right), \nabla T_{k}(u) \chi_{s}\right)\left(\nabla T_{k}\left(u_{n}\right)-\nabla T_{k}(u) \chi_{s}\right) d x \\
=\int_{\Omega \backslash \Omega_{s}} a\left(x, T_{k}(u), 0\right) \nabla T_{k}(u) d x,
\end{gathered}
$$

which implies that

$$
\begin{aligned}
& \int_{\Omega}\left[a\left(x, T_{k}(u), \nabla T_{k}(u)\right)\left(\nabla T_{k}(u)-\nabla v_{0}\right)+\delta(x)\right] d x \\
& \leq \liminf _{n \rightarrow+\infty} \int_{\Omega}\left[a\left(x, T_{k}\left(u_{n}\right), \nabla T_{k}\left(u_{n}\right)\right)\left(\nabla T_{k}\left(u_{n}\right)-\nabla v_{0}\right)+\delta(x)\right] d x \\
& \leq \limsup _{n \rightarrow+\infty} \int_{\Omega}\left[a\left(x, T_{k}\left(u_{n}\right), \nabla T_{k}\left(u_{n}\right)\right)\left(\nabla T_{k}\left(u_{n}\right)-\nabla v_{0}\right)+\delta(x)\right] d x \\
& \leq \int_{\Omega}\left[a\left(x, T_{k}(u), \nabla T_{k}(u)\right)\left(\nabla T_{k}(u) \chi_{s}-\nabla v_{0}\right)+\delta(x)\right] d x \\
& \quad+2 \int_{\Omega \backslash \Omega_{s}} l_{k} \nabla T_{k}(u) \varphi_{k}^{\prime}(0) d x \\
& \quad+2 \int_{\Omega \backslash \Omega_{s}} a\left(x, T_{k}(u), 0\right) \nabla T_{k}(u) \varphi_{k}^{\prime}(0) d x+\int_{\Omega \backslash \Omega_{s}} a\left(x, T_{k}(u), 0\right) \nabla T_{k}(u) d x .
\end{aligned}
$$


Using the fact that $\left[a\left(x, T_{k}(u), \nabla T_{k}(u)\right)\left(\nabla T_{k}(u) \chi_{s}-\nabla v_{0}\right)+\delta(x)\right], l_{k} \nabla T_{k}(u) \varphi_{k}^{\prime}(0), a(x$, $\left.T_{k}(u), 0\right) \nabla T_{k}(u) \varphi_{k}^{\prime}(0)$ and $a\left(x, T_{k}(u), 0\right) \nabla T_{k}(u)$ in $L^{1}(\Omega)$ and letting $s \rightarrow+\infty$, we get, since meas $\left(\Omega \backslash \Omega_{s}\right) \rightarrow 0$,

$$
\begin{aligned}
& \int_{\Omega}\left[a\left(x, T_{k}(u), \nabla T_{k}(u)\right)\left(\nabla T_{k}(u)-\nabla v_{0}\right)+\delta(x)\right] d x \\
& \quad \leq \liminf _{n \rightarrow+\infty} \int_{\Omega}\left[a\left(x, T_{k}\left(u_{n}\right), \nabla T_{k}\left(u_{n}\right)\right)\left(\nabla T_{k}\left(u_{n}\right)-\nabla v_{0}\right)+\delta(x)\right] d x \\
& \quad \leq \limsup _{n \rightarrow+\infty} \int_{\Omega}\left[a\left(x, T_{k}\left(u_{n}\right), \nabla T_{k}\left(u_{n}\right)\right)\left(\nabla T_{k}\left(u_{n}\right)-\nabla v_{0}\right)+\delta(x)\right] d x \\
& \quad \leq \int_{\Omega}\left[a\left(x, T_{k}(u), \nabla T_{k}(u)\right)\left(\nabla T_{k}(u)-\nabla v_{0}\right)+\delta(x)\right] d x .
\end{aligned}
$$

Finally, we have

$$
\begin{gathered}
\lim _{n \rightarrow+\infty} \int_{\Omega}\left[a\left(x, T_{k}\left(u_{n}\right), \nabla T_{k}\left(u_{n}\right)\right)\left(\nabla T_{k}\left(u_{n}\right)-\nabla v_{0}\right)+\delta(x)\right] d x \\
=\int_{\Omega}\left[a\left(x, T_{k}(u), \nabla T_{k}(u)\right)\left(\nabla T_{k}(u)-\nabla v_{0}\right)+\delta(x)\right] d x
\end{gathered}
$$

and by using $\left(A_{3}\right)$, one obtains, by Lemma 2.4

$$
M\left(\nabla T_{k}\left(u_{n}\right)\right) \longrightarrow M\left(\nabla T_{k}(u)\right) \quad \text { in } L^{1}(\Omega) .
$$

Step 6. Equi-integrability of the nonlinearities.

We need to prove that

$$
g_{n}\left(x, u_{n}, \nabla u_{n}\right) \longrightarrow g(x, u, \nabla u) \quad \text { strongly in } L^{1}(\Omega),
$$

in particular it is enough to prove the equi-integrable of $g_{n}\left(x, u_{n}, \nabla u_{n}\right)$. To this purpose, we take $u_{n}-T_{1}\left(u_{n}-v_{0}-T_{h}\left(u_{n}-v_{0}\right)\right)$ as test function in $\left(P_{n}\right)$, we obtain

$$
\begin{aligned}
\int_{\left\{\left|u_{n}-v_{0}\right|>h+1\right\}}\left|g_{n}\left(x, u_{n}, \nabla u_{n}\right)\right| d x & \leq\left\langle f, T_{1}\left(u_{n}-v_{0}-T_{h}\left(u_{n}-v_{0}\right)\right)\right\rangle+\int_{\left\{\left|u_{n}-v_{0}\right|>h\right\}} \delta(x) d x \\
& \leq \int_{\left\{\left|u_{n}-v_{0}\right|>h\right\}}\left(\left|f_{0}\right|+\delta\right)(x) d x+\left.C|| F \chi_{\left\{\left|u_{n}-v_{0}\right|>h\right\}}\right|_{\bar{M} .}
\end{aligned}
$$

Since $\left|f_{0}\right|+\delta \in L^{1}(\Omega), F \in E_{\bar{M}}(\Omega)$, using [14, Lemma 4.16], for all $\varepsilon>0$, then there exists $h(\varepsilon) \geq 1$ such that

$$
\int_{\left\{\left|u_{n}-v_{0}\right|>h(\varepsilon)\right\}}\left|g\left(x, u_{n}, \nabla u_{n}\right)\right| d x<\varepsilon / 2 .
$$

For any measurable subset $E \subset \Omega$, we have

$$
\begin{aligned}
\int_{E}\left|g_{n}\left(x, u_{n}, \nabla u_{n}\right)\right| d x \leq & \int_{E} b(h(\varepsilon))\left(c(x)+M\left(\nabla T_{h(\varepsilon)}\left(u_{n}\right)\right)\right) d x \\
& +\int_{\left\{\left|u_{n}\right|>h(\varepsilon)\right\}}\left|g\left(x, u_{n}, \nabla u_{n}\right)\right| d x .
\end{aligned}
$$


In view of (4.65) there exists $\eta(\varepsilon)>0$ such that

$$
\int_{E} b(h(\varepsilon))\left(c(x)+M\left(\nabla T_{h(\varepsilon)}\left(u_{n}\right)\right)\right) d x<\varepsilon / 2 \quad \forall E \text { such that }|E|<\eta(\varepsilon) .
$$

Finally, combining (4.75) and (4.76), one easily has

$$
\int_{E}\left|g_{n}\left(x, u_{n}, \nabla u_{n}\right)\right| d x<\varepsilon \quad \forall E \text { such that }|E|<\eta(\varepsilon),
$$

which implies (4.66).

Moreover, if we take $v_{0}$ as function test in $\left(P_{n}\right)$, we get

$$
\int_{\Omega} g_{n}\left(x, u_{n}, \nabla u_{n}\right) u_{n} d x \leq \int_{\Omega} \delta(x)+d x+\int_{\Omega} g_{n}\left(x, u_{n}, \nabla u_{n}\right) v_{0} d x+\left\langle f, u_{n}-v_{0}\right\rangle,
$$

hence

$$
\int_{\Omega} g_{n}\left(x, u_{n}, \nabla u_{n}\right) u_{n} d x \leq \beta,
$$

where $\beta$ is some positive constant, then by using Fatou's lemma, we have

$$
g(x, u, \nabla u) u \in L^{1}(\Omega) .
$$

Step 7. Passing to the limit.

We take $v \in K_{\psi} \cap W_{0}^{1} E_{M}(\Omega) \cap L^{\infty}(\Omega)$, in $\left(P_{n}\right)$, we can write

$$
\int_{\Omega} a\left(x, u_{n}, \nabla u_{n}\right) \nabla\left(u_{n}-v\right) d x+\int_{\Omega} g\left(x, u_{n}, \nabla u_{n}\right)\left(u_{n}-v\right) d x \leq\left\langle f, u_{n}-v\right\rangle,
$$

which implies that

$$
\begin{gathered}
\int_{\Omega} a\left(x, u_{n}, \nabla u_{n}\right) \nabla\left(u_{n}-v_{0}\right) d x+\int_{\Omega} a\left(x, u_{n}, \nabla u_{n}\right) \nabla\left(v_{0}-v\right) d x \\
+\int_{\Omega} g\left(x, u_{n}, \nabla u_{n}\right)\left(u_{n}-v\right) d x \leq\left\langle f, u_{n}-v\right\rangle .
\end{gathered}
$$

By Fatou's lemma and the fact that

$$
a\left(x, u_{n}, \nabla u_{n}\right) \rightarrow a(x, u, \nabla u)
$$

weakly in $\left(L_{\bar{M}}(\Omega)\right)^{N}$ for $\sigma\left(\Pi L_{\bar{M}}, \Pi E_{M}\right)$ one easily sees that

$$
\begin{gathered}
\int_{\Omega} a(x, u, \nabla u) \nabla\left(u-v_{0}\right) d x+\int_{\Omega} a(x, u, \nabla u) \nabla\left(v_{0}-v\right) d x \\
+\int_{\Omega} g(x, u, \nabla u)(u-v) d x \leq\langle f, u-v\rangle .
\end{gathered}
$$

Hence

$$
\int_{\Omega} a(x, u, \nabla u) \nabla(u-v) d x+\int_{\Omega} g(x, u, \nabla u)(u-v) d x \leq\langle f, u-v\rangle .
$$


Now, let $v \in K_{\psi} \cap L^{\infty}(\Omega)$, by the condition ( $\left.\mathrm{A}_{5}\right)$ there exists $v_{j} \in K_{\psi} \cap W_{0}^{1} E_{M}(\Omega) \cap$ $L^{\infty}(\Omega)$ such that $v_{j}$ converges to $v$ modular, let $h>\left\|v_{0}\right\|_{\infty}$, taking $v=T_{h}\left(v_{j}\right)$ in (4.79), we have

$$
\int_{\Omega} a(x, u, \nabla u) \nabla\left(u-T_{h}\left(v_{j}\right)\right) d x+\int_{\Omega} g(x, u, \nabla u)\left(u-T_{h}\left(v_{j}\right)\right) d x \leq\left\langle f, u-T_{h}\left(v_{j}\right)\right\rangle .
$$

We can easily pass to the limit as $j \rightarrow+\infty$ to get

$$
\begin{gathered}
\int_{\Omega} a(x, u, \nabla u) \nabla\left(u-T_{h}(v)\right) d x+\int_{\Omega} g(x, u, \nabla u)\left(u-T_{h}(v)\right) d x \\
\leq\left\langle f, u-T_{h}(v)\right\rangle \quad \forall v \in K_{\psi} \cap L^{\infty}(\Omega),
\end{gathered}
$$

the same, we pass to the limit as $h \rightarrow+\infty$, we deduce

$$
\int_{\Omega} a(x, u, \nabla u) \nabla(u-v) d x+\int_{\Omega} g(x, u, \nabla u)(u-v) d x \leq\langle f, u-v\rangle \quad \forall v \in K_{\psi} \cap L^{\infty}(\Omega) .
$$

This completes the proof of the theorem.

\section{References}

[1] R. Adams, Sobolev Espaces, Academic Press, New York, 1975.

[2] A. Benkirane, Approximations de type Hedberg dans les espaces $W^{m} L \log L(\Omega)$ et applications [Hedberg-type approximations in the spaces $W^{m} L \log L(\Omega)$ and applications], Toulouse. Faculté des Sciences. Annales. Mathématiques. Série 511 (1990), no. 2, 67-78 (French).

[3] A. Benkirane and A. Elmahi, An existence theorem for a strongly nonlinear elliptic problem in Orlicz spaces, Nonlinear Analysis. Theory, Methods \& Applications. An International Multidisciplinary Journal. Series A: Theory and Methods 36 (1999), no. 1, 11-24.

[4] - A strongly nonlinear elliptic equation having natural growth terms and $L^{1}$ data, Nonlinear Analysis. Theory, Methods \& Applications. An International Multidisciplinary Journal. Series A: Theory and Methods 39 (2000), no. 4, 403-411.

[5] A. Benkirane, A. Elmahi, and D. Meskine, An existence theorem for a class of elliptic problems in $L^{1}$, Applicationes Mathematicae 29 (2002), no. 4, 439-457.

[6] A. Benkirane and J.-P. Gossez, An approximation theorem in higher order Orlicz-Sobolev spaces and applications, Polska Akademia Nauk. Instytut Matematyczny. Studia Mathematica 92 (1989), no. 3, 231-255.

[7] A. Bensoussan, L. Boccardo, and F. Murat, On a nonlinear partial differential equation having natural growth terms and unbounded solution, Annales de l'Institut Henri Poincaré. Analyse Non Linéaire 5 (1988), no. 4, 347-364.

[8] A. Elmahi and D. Meskine, Existence of solutions for elliptic equations having natural growth terms in Orlicz spaces, Abstract and Applied Analysis 2004 (2004), no. 12, 1031-1045.

[9] _ Unilateral elliptic problems in $L^{1}$ with natural growth terms, Journal of Nonlinear and Convex Analysis. An International Journal 5 (2004), no. 1, 97-112.

[10] M. Fuchs and L. Gongbao, $L^{\infty}$-bounds for elliptic equations on Orlicz-Sobolev spaces, Archiv der Mathematik. Archives of Mathematics. Archives Mathématiques 72 (1999), no. 4, 293-297.

[11] M. Fuchs and G. Seregin, A regularity theory for variational integrals with L $\ln L$-growth, Calculus of Variations and Partial Differential Equations 6 (1998), no. 2, 171-187. 
[12] _ Regurality for solutions of variational problems in the deformation theory of plasticity with logarithmic hardening, Proceedings of St. Petersburg Mathematical Society, Vol. 5 (Bonn, 1995), 1998, pp. 184-222, English translation in American Mathematical Society Translations, Series 2. preprint no. 421, SFB256.

[13] _ Variational methods for fluids of Prandtl-Eyring type and plastic materials with logarithmic hardening, Mathematical Methods in the Applied Sciences 22 (1999), no. 4, 317-351.

[14] J.-P. Gossez, Nonlinear elliptic boundary value problems for equations with rapidly (or slowly) increasing coefficients, Transactions of the American Mathematical Society 190 (1974), 163-205.

[15] _ A strongly nonlinear elliptic problem in Orlicz-Sobolev spaces, Nonlinear Functional Analysis and Its Applications (Berkeley, Calif, 1983), Proc. Sympos. Pure Math., vol. 45, American Mathematical Society, Rhode Island, 1986, pp. 455-462.

[16] J.-P. Gossez and V. Mustonen, Variational inequalities in Orlicz-Sobolev spaces, Nonlinear Analysis. Theory, Methods \& Applications. An International Multidisciplinary Journal. Series A: Theory and Methods 11 (1987), no. 3, 379-392.

[17] M. A. Krasnosel'skii and Y. B. Rutikii, Convex Functions and Orlicz Spaces, P. Noordhoff, Groningen, 1969.

[18] A. Porretta, Existence for elliptic equations in $L^{1}$ having lower order terms with natural growth, Portugaliae Mathematica 57 (2000), no. 2, 179-190.

L. Aharouch: Département de Mathématiques et Informatique, Faculté des Sciences Dhar-Mahraz, Université Sidi Mohamed Ben Abdallah B.P. 1796, Atlas Fès, Morocco

E-mail address: laharouch@fsdmfes.ac.ma

A. Benkirane: Département de Mathématiques et Informatique, Faculté des Sciences Dhar-Mahraz, Université Sidi Mohamed Ben Abdallah B.P. 1796, Atlas Fès, Morocco

E-mail address: abdelmoujib@iam.net.ma

M. Rhoudaf: Département de Mathématiques et Informatique, Faculté des Sciences Dhar-Mahraz, Université Sidi Mohamed Ben Abdallah B.P. 1796, Atlas Fès, Morocco

E-mail address: mrhoudaf@fsdmfes.ac.ma 


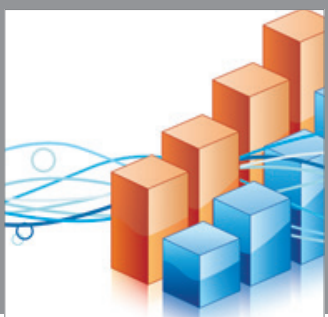

Advances in

Operations Research

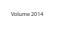

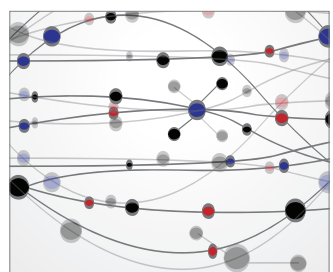

\section{The Scientific} World Journal
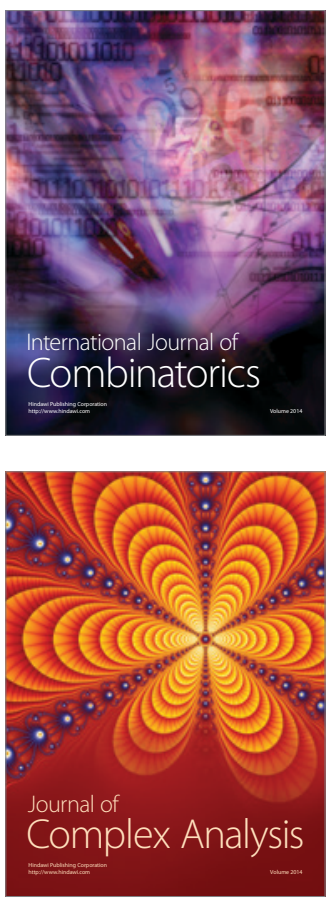

International Journal of

Mathematics and

Mathematical

Sciences
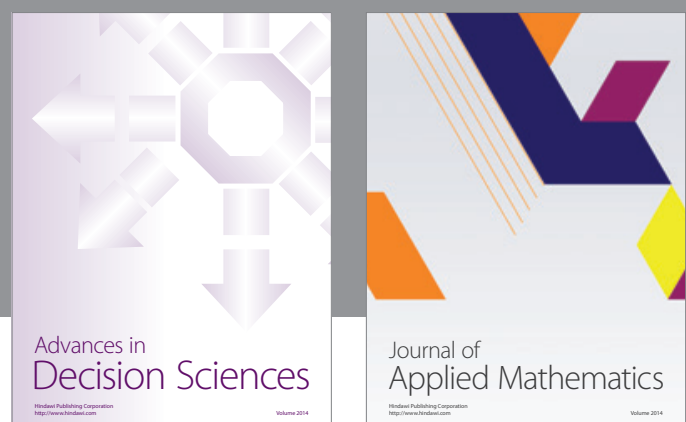

Journal of

Applied Mathematics
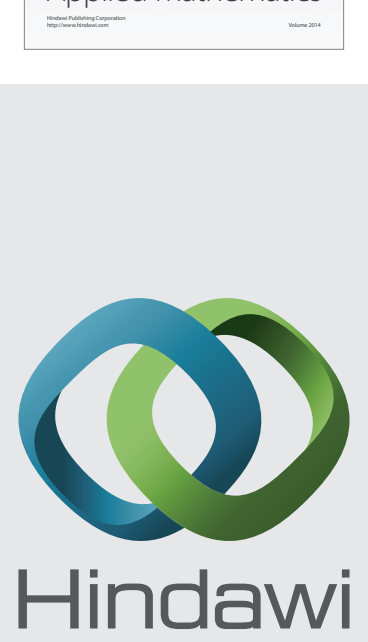

Submit your manuscripts at http://www.hindawi.com
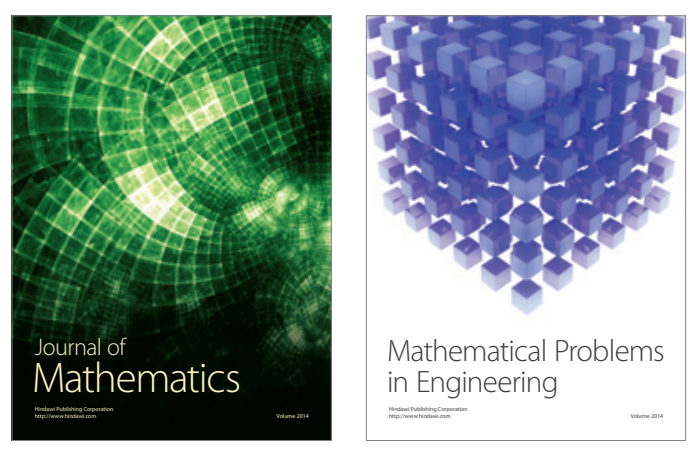

Mathematical Problems in Engineering
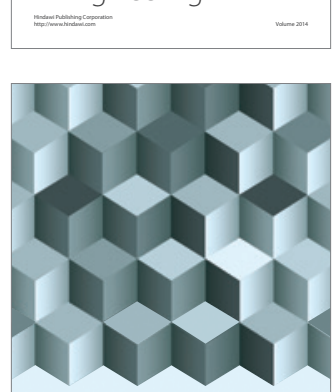

Journal of

Function Spaces
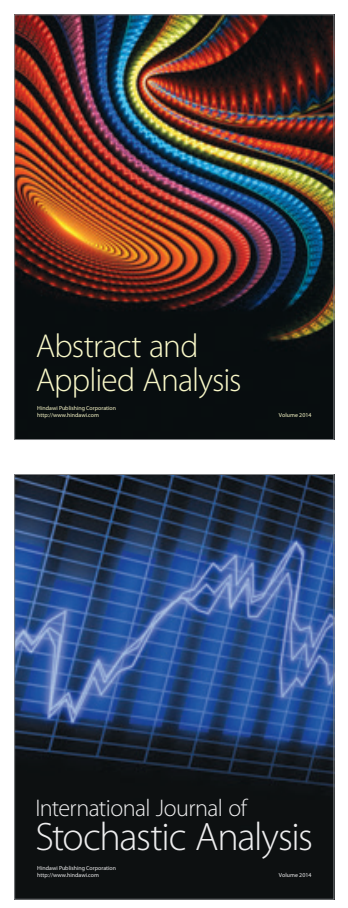

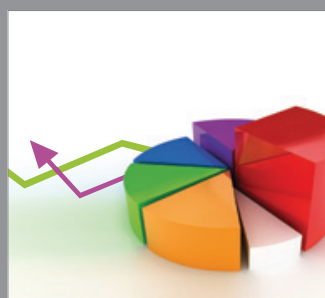

ournal of

Probability and Statistics

Promensencen
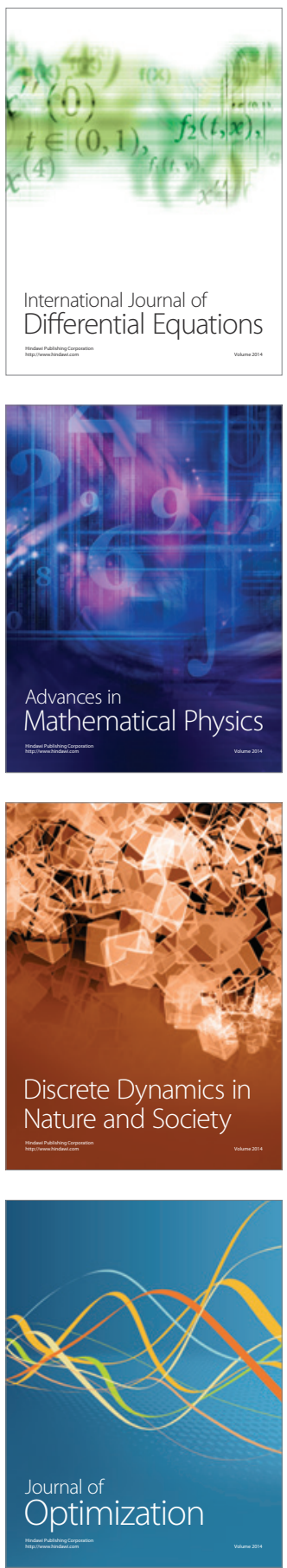